\title{
NUMERICAL SIMULATION OF LOAD TRANSFER IN MOORING ANCHOR SYSTEMS
}

\author{
KARENA Q. JIMENEZ1, JORGE P. TAMAYO², SAMIR MAGHOUS ${ }^{3}$ AND \\ ALEXANDRE L. BRAUN ${ }^{4}$ \\ ${ }^{1}$ Graduate student at Federal University of Rio Grande do Sul (UFRGS) \\ Av. Osvaldo Aranha 99-3 Floor, 90035-190, Porto Alegre, RS, Brazil \\ karenaquiroz@gmail.com and https://www.ufrgs.br/cemacom/ \\ ${ }^{2}$ Professor at Federal University of Rio Grande do Sul (UFRGS)

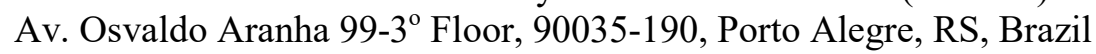 \\ jorge.tamayo@ufrgs.br and https://www.ufrgs.br/cemacom/ \\ ${ }^{3}$ Professor at Federal University of Rio Grande do Sul (UFRGS)

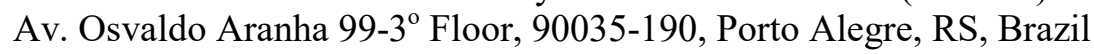 \\ samir.maghous@ufrgs.br and https://www.ufrgs.br/cemacom/ \\ ${ }^{4}$ Professor at Federal University of Rio Grande do Sul (UFRGS) \\ Av. Osvaldo Aranha 99-3 Floor, 90035-190, Porto Alegre, RS, Brazil \\ allbraun@ig.com.br and https://www.ufrgs.br/cemacom/
}

Key words: Embedded Model, Bond-slip Interface Model, Large strains, FEM, Corotational.

\begin{abstract}
A numerical model for the load transfer mechanism in mooring anchor systems, commonly used in offshore petroleum industry, is presented in this work. Special attention is paid to the mechanical modelling of the contact interaction of the two medium components, namely the soil and the embedded mooring line. Resorting to the "embedded element concept" [1], a mixed 3D-1D finite element formulation is developed in the context of finite elastoplasticity. A Tresca-like model is used to describe the nonlinear material behaviour of the surrounding soil under undrained conditions, whereas the embedded mooring line regarded as curvilinear bar-like inclusion is assumed to behave elastically with account for geometric nonlinearities. The Mohr-Coulomb model is employed to define the bond-stress and bond-slip relationships at the interface. Nonlinear static and dynamic analyses are performed with a corotational kinematic description in order to include large deformation in the problem [2]. Preliminary results indicate that the main frequencies of the dynamic load applied to the mooring line-soil system are much lower than those of the system itself, thus the overall system may be evaluated disregarding inertial effects. Further simulations based on parametric studies by varying relevant problem parameters are needed to corroborate this result. Moreover, the average load attenuation induced by friction along the soil/mooring line interface for the studied cases is around $25 \%$. Formulation of the interface constitutive behaviour in the context of large strain to address large relative movements between embedded inclusion and surrounding soil is an ongoing task [3]. Parallel implementation of
\end{abstract}


the finite element model with specific data structure storage and iterative solver is currently addressed to handle large 3D computational models.

\section{INTRODUCTION}

Exploitation of petroleum in oceans is an activity which requires the use of floating platforms at the sea level in the worksite. These platforms, which are subjected to aerodynamic and hydrodynamic loads at their surface, are stabilized by means of mooring line systems composed of mooring lines and anchors. The applied loads at the platform are transferred to the mooring lines through fairlead points in which friction forces develop along the mooring line by contact with the sea water and soil, i.e. the developed load in the anchoring device at the end of the mooring line is different from that at the platform surface. At the end of the trajectory, the anchoring device penetrates the sea bed and fixes the platform by free fall, generating a buried segment. Precisely, the evaluation of friction forces along this segment becomes a crucial issue in this problem, as the overall system depends upon the anchor strength. Similar works about this topic may be found in references $[4,5]$.

In this paper a mixed 3D-1D formulation based on the displacement finite element method is presented to model the buried segment of the mooring line system. Installation effects are not accounted for in the analysis for simplification. Also, it is considered that the mechanical system is in equilibrium at the start of the analysis. In this approach the soil composed of matrix particles is a three-dimensional solid medium, while the buried segment or inclusion is is efficient considering medium. The solid elements with stabilizat However, they may suffer from convergence p Then, the performance of classieal hexahedral

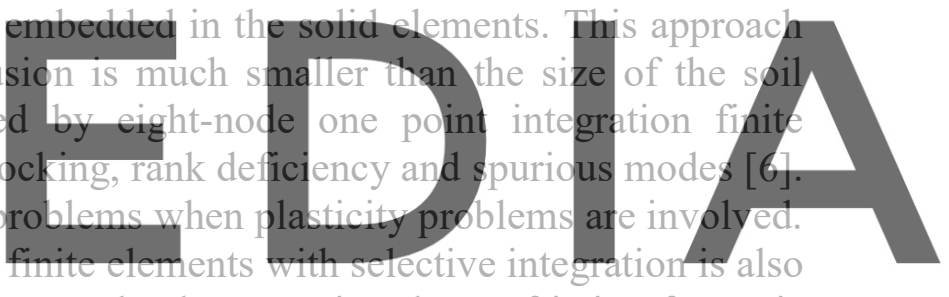
considered for the undrained situation. In all cases, load attenuation due to friction forces is Register fordfrese https//www.scipedia.com to download the version without the watermark

2 FINITE STRAIN APPROACH

\subsection{Corotational description of matrix particles motion and constitutive equation}

A corotational description as shown in Figure 1 is adopted to describe the motion of the continuum body. The reference frame is decomposed into a base configuration, namely the undeformed configuration, and a corotated one attached to the rigid body motion. The coordinate system of the corotated configuration $\hat{\mathbf{x}}$, which is defined at the finite element center, follows the material motion and is related to the global coordinate system by means of a rotation matrix. Note that rotation $\mathbf{R}$ transforming the current configuration into the corotational configuration is defined by the rotation component in polar decomposition of deformation gradient. In this manner, the strain rate tensor in the corotational system may be defined as: 


$$
\hat{\mathbf{d}}_{\mathrm{m}}=\frac{1}{2}\left[\frac{\partial \hat{\mathbf{v}}_{\mathrm{m}}^{\mathrm{def}}}{\partial \hat{\mathbf{x}}}+\left(\frac{\partial \hat{\mathbf{v}}_{\mathrm{m}}^{\mathrm{def}}}{\partial \hat{\mathbf{x}}}\right)^{\mathrm{T}}\right]
$$

where subscript $\mathrm{m}$ refers to matrix particles and $\hat{\mathbf{v}}_{\mathrm{m}}^{\mathrm{def}}$ represents the velocity field associated to the deformation part of the motion according to the corotational system. Considering that all kinematics variables are known at configuration $t=t_{n}$, the displacement field can be obtained from integration of the strain rate tensor in the time interval $\left[\mathrm{t}_{\mathrm{n}}, \mathrm{t}_{\mathrm{n}+1}\right]$.

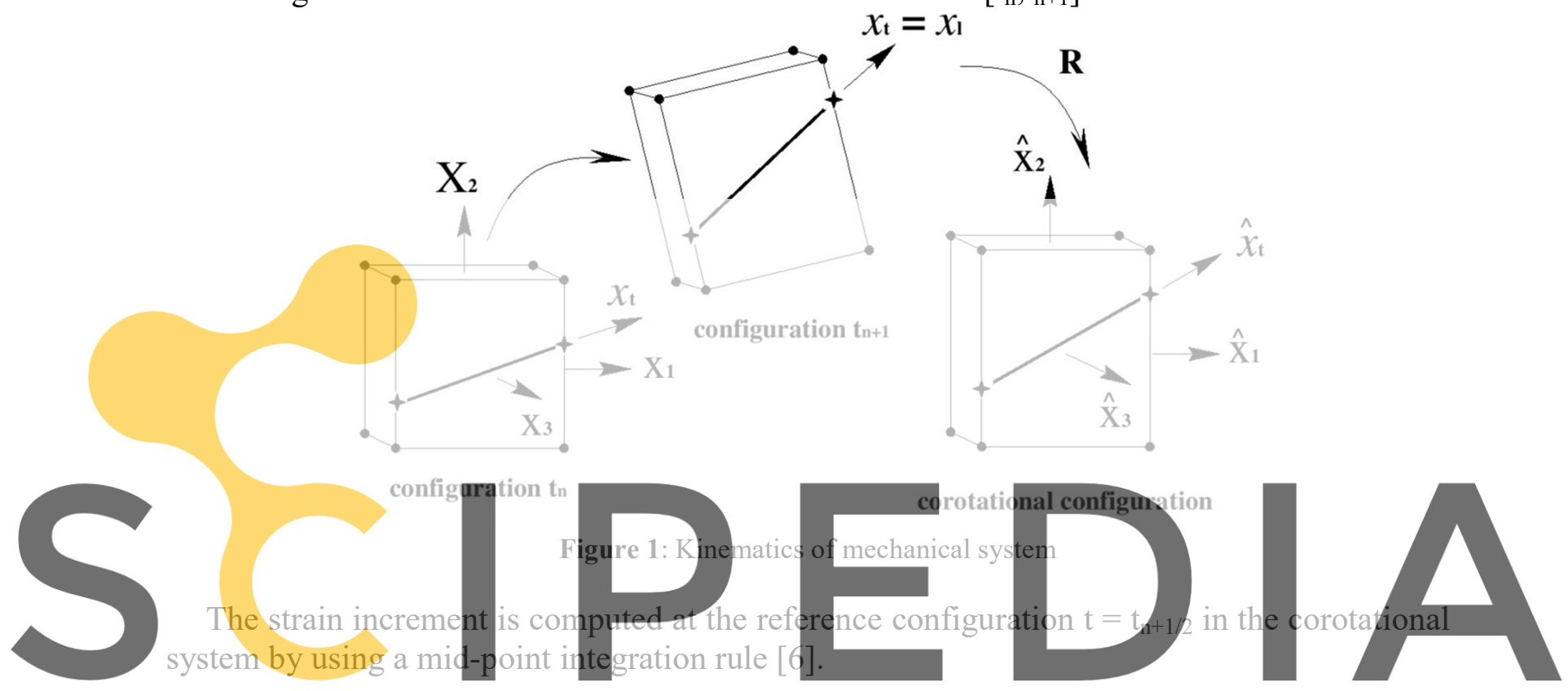

Register for free at https//www.\$ॄ⿺

where $\Delta \hat{\mathbf{u}}_{\mathrm{m}}^{\mathrm{def}}$ and $\hat{\mathbf{x}}_{\mathrm{n}+1 / 2}$ represent the deformation part of the displacement increment $\Delta \hat{\mathbf{u}}_{\mathrm{m}}$ and the intermediate geometric configuration in the corotational system, respectively. The latter is defined in the following manner:

$$
\hat{\mathbf{x}}_{\mathrm{n}+1 / 2}=\mathbf{R}_{\mathrm{n}+1 / 2} \cdot \mathbf{x}_{\mathrm{n}+1 / 2}=\frac{1}{2} \mathbf{R}_{\mathrm{n}+1 / 2} \cdot\left(\mathbf{x}_{\mathrm{n}}+\mathbf{x}_{\mathrm{n}+1}\right)
$$

where $\mathbf{R}_{n+1 / 2}$ is an orthogonal matrix, which performs rotation from the global to the corotational coordinate system at element level. The global displacement increment $\Delta \mathbf{u}_{\mathrm{m}}$ in time interval $\left[\mathrm{t}_{\mathrm{n}}, \mathrm{t}_{\mathrm{n}+1}\right]$ is decomposed as:

$$
\Delta \mathbf{u}_{\mathrm{m}}=\Delta \mathbf{u}_{\mathrm{m}}^{\mathrm{def}}+\Delta \mathbf{u}_{\mathrm{m}}^{\mathrm{rot}}
$$

with $\Delta \mathbf{u}_{\mathrm{m}}^{\text {def }}$ and $\Delta \mathbf{u}_{\mathrm{m}}^{\text {rot }}$ denoting the deformation and rotation parts of the displacement increment. Meanwhile, the same quantity expressed in the corotational system is defined as: 


$$
\Delta \hat{\mathbf{u}}_{\mathrm{m}}^{\mathrm{def}}=\hat{\mathbf{x}}_{\mathrm{n}+1}-\hat{\mathbf{x}}_{\mathrm{n}}=\mathbf{R}_{\mathrm{n}+1 / 2} \cdot \Delta \mathbf{u}_{\mathrm{m}}^{\mathrm{def}}
$$

in which subscripts $n$ and $n+1$ refer to geometric configurations at times $t_{n}$ and $t_{n+1}$, respectively. Omitting time subscripts, the rotation matrix can be expressed as:

$$
\mathbf{R}_{1 j}=\frac{\mathbf{r}_{1 j}}{\mathbf{r}_{1}^{\mathrm{T}} \mathbf{r}_{1}} ; \quad \mathbf{R}_{2 \mathrm{j}}=\frac{\left(\mathbf{r}_{2 \mathrm{j}}+\mathbf{r}_{\mathrm{cj}}\right)}{\left(\mathbf{r}_{2 \mathrm{j}}+\mathbf{r}_{\mathrm{cj}}\right)^{\mathrm{T}}\left(\mathbf{r}_{2 \mathrm{j}}+\mathbf{r}_{\mathrm{cj}}\right)} \quad ; \quad \mathbf{R}_{3 \mathrm{j}}=\frac{\mathbf{r}_{3 \mathrm{j}}}{\mathbf{r}_{3}^{\mathrm{T}} \mathbf{r}_{3}} \quad(\mathrm{j}=1,2,3)
$$

with,

$$
\mathbf{r}_{1 \mathrm{j}}=\xi^{\mathrm{T}} \mathbf{x}_{\mathrm{j}} \quad ; \quad \mathbf{r}_{2 \mathrm{j}}=\eta^{\mathrm{T}} \mathbf{x}_{\mathrm{j}} \quad ; \quad \mathbf{r}_{\mathrm{cj}}=-\frac{\mathbf{r}_{1 \mathrm{j}}^{\mathrm{T}} \mathbf{r}_{2 \mathrm{j}}}{\mathbf{r}_{1 \mathrm{j}}^{\mathrm{T}} \mathbf{r}_{1 \mathrm{j}}} \mathbf{r}_{1 \mathrm{j}} \quad ; \quad \mathbf{r}_{3 \mathrm{j}}=\mathbf{r}_{1 \mathrm{j}} \times\left(\mathbf{r}_{2 \mathrm{j}}+\mathbf{r}_{\mathrm{cj}}\right)
$$

where $(\xi, \eta, \zeta)$ and $\mathbf{x}$ are respectively vectors defining the natural and global nodal coordinates of the current element [2]. Otherwise, the state equation which relates a rotational time derivate of stress tensor to the strain rate tensor is defined in the following manner:

$$
\dot{\hat{\sigma}}_{\mathrm{m}}^{\mathrm{J}}=\hat{\mathbf{D}}^{\mathrm{e}} \cdot\left(\hat{\mathbf{d}}_{\mathrm{m}}-\hat{\mathbf{d}}_{\mathrm{m}}^{\mathrm{p}}\right) \text { with } \quad \dot{\hat{\sigma}}_{\mathrm{m}}^{\mathrm{J}}=\dot{\hat{\sigma}}_{\mathrm{m}}+\hat{\sigma}_{\mathrm{m}} \cdot \hat{\Omega}_{\mathrm{m}}-\hat{\Omega}_{\mathrm{m}} \cdot \hat{\sigma}_{\mathrm{m}}
$$

in which $\hat{\mathbf{d}}_{\mathrm{m}}^{\mathrm{p}}, \hat{\Omega}_{\mathrm{m}}$ and $\hat{\sigma}_{\mathrm{m}}$ denotes the plastic strain rate tensor, spin tensor and Cauchy stress tensor in the corotational system, respectively. Meanwhile, $\widehat{\mathbb{D}}^{e}$ could represent the fourthorder elastic or elastic-plastic constitutive ten or of the matrix. The aforementioned equation is integrated within the time interval in the context of large strains.

2.2 Description of deformation in embedded inclusion and constitudive equation

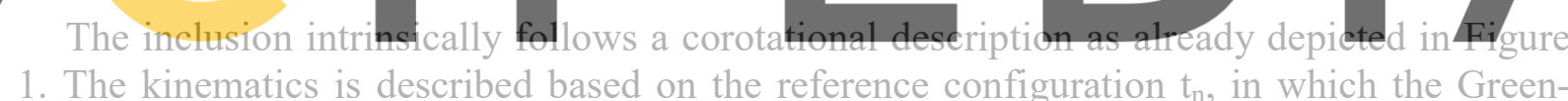

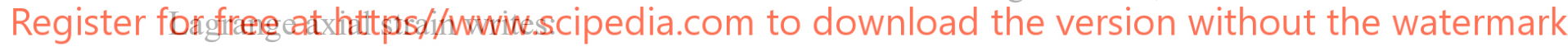

$$
\tilde{\mho}_{\mathrm{c}}=\frac{\partial u_{c}}{\partial x_{t}}+\frac{1}{2}\left[\frac{\partial \mathbf{u}_{\mathrm{c}}}{\partial x_{t}}\right]^{\mathrm{T}}\left[\frac{\partial \mathbf{u}_{\mathrm{c}}}{\partial x_{t}}\right] \quad \text { with } u_{c}=\mathbf{u}_{\mathrm{c}} \cdot \mathbf{I}
$$

where $\mathbf{u}_{\mathrm{c}}$ is the displacement vector at any point along the embedded inclusion, $x_{t}$ refers to the current tangent direction of the inclusion as illustrated in Figure 1, and $\mathbf{l}$ is the unit vector along the inclusion. The displacement vector $\mathbf{u}_{\mathrm{c}}$ may be written in terms of the relative tangential displacement $u_{\mathrm{s}}$, and the displacement components in the global coordinate system of the geometrically coinciding matrix particle $\mathbf{u}_{m}$ as follows:

$$
\mathbf{u}_{\mathrm{c}}-\mathbf{u}_{m}=u_{s} \mathbf{l}
$$

Otherwise, it is assumed an elastic behavior for the inclusion in which the stress increment of second Piola-Kirchhoff stress $\Delta \pi$ during the time interval $\left[\mathrm{t}_{\mathrm{n}}, \mathrm{t}_{\mathrm{n}+1}\right]$ is related to axial strain by means of the elastic stiffness of the inclusion $E_{\mathrm{c}}$.

$$
\Delta \pi=E_{c} \mathcal{E}_{\mathrm{c}}
$$




\subsection{Constitutive behavior of the interface}

The kinematics of the inclusion is better described in its local coordinate system in terms of tangential and normal relative displacement components. Particularly, the relative displacement in the normal direction is zero due to assumption of bond-slip at the interface. The Mohr-Coulomb yield criterion is then used to separate elastic and elastic-plastic stresses. The incremental form of the constitutive equations at the interface in the elastic regime is given by the following expression:

$$
\Delta \tau_{\text {int }}=k_{s} \Delta u_{s}
$$

While in the plastic regime it is written as:

$$
\Delta \tau_{\text {int }}=\frac{k_{s}}{1+\frac{k_{s}}{k_{n} \operatorname{tg} \phi \operatorname{tg} \psi+h \operatorname{sign}\left(\tau_{\text {int }}\right)}} \Delta u_{s}
$$

in which $\phi$ and $\psi$ are respectively the friction and dilatation angles of the interface and $h$ is the hardening modulus of cohesion. Meanwhile, $k_{n}$ and $k_{s}$ refer to normal and tangential stiffness moduli in force per cubic length. The aforementioned equation is obtained in [6] by applying the consistency condition and forcing a null component of displacement in the normal direction.
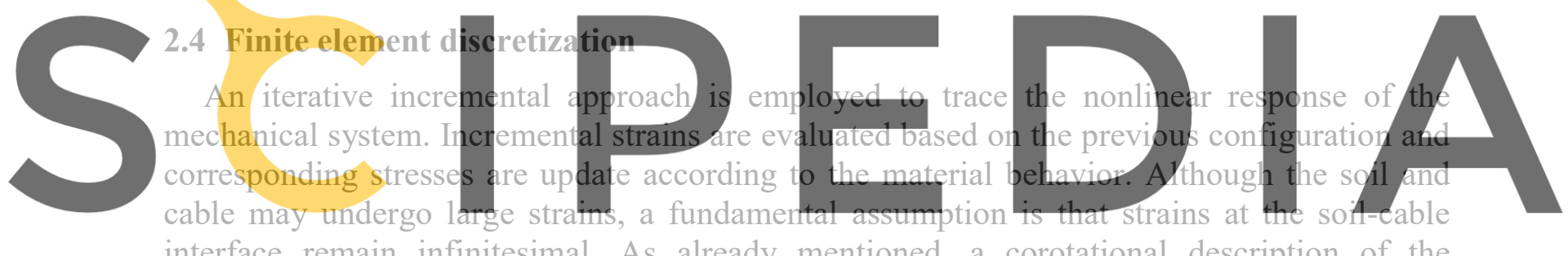
interface remain infinitesimal. As already mentioned, a corotational description of the

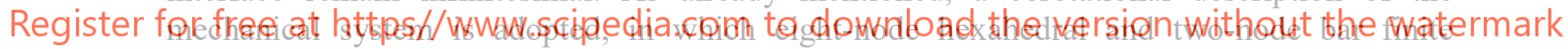
elements are used respectively to represent the soil and cable. The unknown variables of the problem are the global vector of nodal displacements of the soil $\mathbf{u}_{\mathrm{s}}$ and relative displacements of the cable elements $\mathbf{w}_{\mathrm{t}}$ in the tangential direction. In this manner, the total displacement of the cable is recovered by adding these two variables in the global coordinate system. By using the principle of virtual work and applying the finite element method, the following coupled equation is obtained at the element level.

$$
\left[\begin{array}{ll}
\boldsymbol{k}_{u u} & \boldsymbol{k}_{u w} \\
\boldsymbol{k}_{w u} & \boldsymbol{k}_{w w}
\end{array}\right]\left[\begin{array}{l}
\mathbf{u}_{s} \\
\mathbf{w}_{t}
\end{array}\right]=\left[\begin{array}{l}
\boldsymbol{p}_{t}-\boldsymbol{q}_{u u} \\
\boldsymbol{r}_{t}-\boldsymbol{q}_{w w}
\end{array}\right]
$$

in which $\boldsymbol{k}_{u w}$ and $\boldsymbol{k}_{w u}$ are coupled matrices of soil and cable, $\boldsymbol{k}_{u u}$ contains the cable and soil contributions and matrix $\boldsymbol{k}_{w w}$ contains the cable and interface rigidities. Variables $\boldsymbol{p}_{t}$ and $\boldsymbol{r}_{t}$ are respectively the nodal external forces of soil and cable, while $\boldsymbol{q}_{u u}$ and $\boldsymbol{q}_{w w}$ refer to corresponding internal nodal forces. The explicit expressions of these matrices can be found in reference [6] and the reader is referred to that work for details . After assembling all element contributions, the set of equations of the incremental form of the nonlinear problem of the coupled system is written in the following form: 


$$
\mathbf{K}^{\tan }(\mathbf{U}) \cdot \mathbf{U}=\mathbf{F}^{\mathrm{ext}}-\mathbf{F}^{\mathrm{int}}(\mathbf{U})
$$

where $\mathbf{K}^{\mathrm{tan}}$ is the global tangent stiffness matrix related to left hand side of Equation (14), $\mathbf{F}^{\mathrm{ext}}$ and $\mathbf{F}^{\text {int }}$ are the global external and internal force vectors, respectively. The unknown incremental displacement $\mathbf{U}$ contains the increments of displacements of soil $\Delta \mathbf{u}_{\mathrm{s}}$ and relative displacement at the interface $\Delta \mathbf{w}_{\mathrm{t}}$.

\section{NUMERICAL APPLICATION}

\subsection{Problem description}

A typical mooring system as depicted in Figure 2 is composed of a mooring line and a torpedo anchor. The mooring line has a suspended part in the seawater and a buried segment below the sea bed. As it may be seen, the buried segment joins the point on the seabed surface with that of the torpedo position. Torpedo anchors are introduced into the seabed by free fall from the platform, penetrating $30 \mathrm{~m}$ to $150 \mathrm{~m}$ into the soil. The holding capacity of the torpedo is then established with soil properties and penetration depth. In this context, the numerical simulation is restricted to the study of the embedded part in which load $\mathbf{f}_{\mathrm{b}}$ is applied at the upper end of the cable. This load is known in advance since load record $\mathbf{F}_{\mathrm{p}}$ is monitored at the platform. The focus of the study is to determine load $\mathbf{f}_{\mathrm{t}}$ acting at the anchoring device after attenuation due to friction forces at the soil-cable interface. Load $\mathbf{f}_{t}$ is an important issue

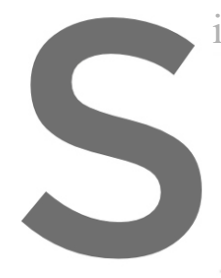
in design of anchors 1
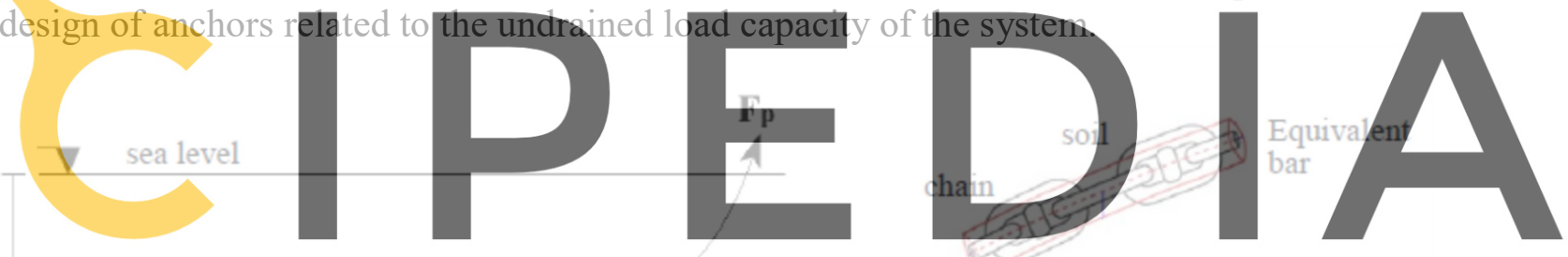

Register for free at htțs//www.scipedia.com to download the version without the watermark

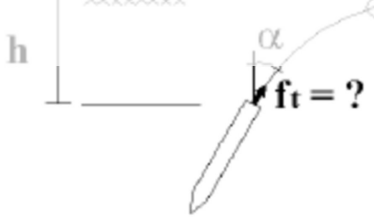

Figure 2: Schematic representation of load transfer along mooring line and equivalent bar modeling of buried segment

The geometry and finite element model used after a mesh sensitivity analysis are shown in Figure 3. This is a parallelepiped volume of soil ( $15 \mathrm{~m}$ height, $40 \mathrm{~m}$ length and $7 \mathrm{~m}$ width) whose upper face lies on the seabed plane $y=0$ in which the load $\mathbf{f}_{\mathrm{b}}$ is applied at the cable. The dimensions are established in order to avoid the influence of the boundary conditions over the region of interest. The shape of the embedded mooring line has an inverse catenary form where an equivalent circular cross section is assumed to represent the mooring line made up of chains. The mooring line is located at the vertical mid-plane of the domain in which various cable segments are automatically defined according to mesh discretization. Smoothwall boundary conditions are imposed on the bottom and lateral surfaces of the computational 
domain. A hydrostatic load $\mathbf{q}_{\mathrm{w}}$ representing the pressure exerted by the seawater $(\mathrm{H}=2135 \mathrm{~m})$ is applied on the top surface. The anchoring point at the end of the embedded mooring line is considered to be fixed, indicating that the torpedo is sufficiently rigid to constraint all degrees of freedom at this point.

The mechanical response of the soil-mooring cable system under undrained loading conditions is investigated in the context of total stress constitutive behavior. The soil is assumed to be elastic and perfect plastic material following a Tresca-like model in which the undrained shear strength and Young modulus increase linearly with depth. The mooring line is considered to be elastic with an axial rigidity defined by the product of its equivalent area and Young modulus. The elastic-plastic behavior of the soil-cable interface is defined by normal and tangential stiffness moduli $k_{n}$ and $k_{s}$, cohesion $\mathrm{c}$, friction angle $\phi$ and dilatancy angle $\psi$. Table 1 summarizes all material properties.

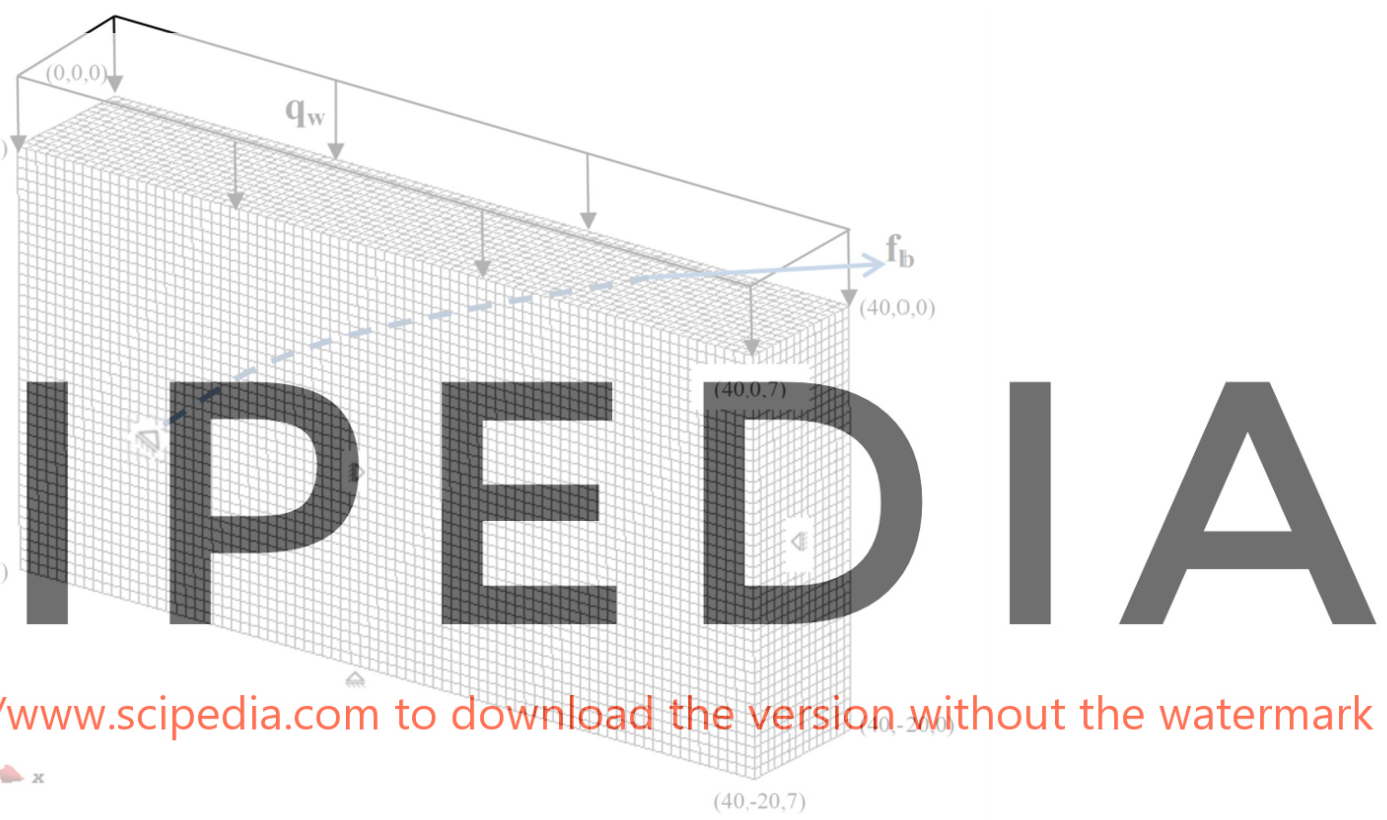

Figure 3: Finite element mesh corresponding to the anchoring point at $\mathrm{h}=15 \mathrm{~m}$ depth.

Table 1: Mechanical properties

\begin{tabular}{lll}
\hline Soil & Undrained shear strength $\mathrm{S}_{\mathrm{u}}(\mathrm{y})=\alpha \mathrm{y}$ & $\alpha=1.4 \mathrm{kPa} / \mathrm{m}$ \\
\hline & Elastic modulus $\mathrm{E}(\mathrm{y})=\beta \mathrm{S}_{\mathrm{u}}(\mathrm{y})$ & $\beta=300$ \\
\hline & Poisson ratio & $v=0.495$ \\
\hline Saturated specific weight & $\gamma=15.1 \mathrm{kN} / \mathrm{m}^{3}$ \\
\hline Mooring cable & Equivalent bar diameter & $\mathrm{d}_{\mathrm{c}}=0.24 \mathrm{~m}$ \\
\hline & Axial rigidity & $\mathrm{E}_{\mathrm{c}} \mathrm{A}_{\mathrm{c}}=1.1 \times 10^{6} \mathrm{kN}$ \\
\hline & Cohesion & $\mathrm{c}=0 \mathrm{kPa}$ \\
\hline & Internal friction angle & $\phi=30^{\circ}$ \\
\hline & Dilatancy angle & $\psi=0^{\circ}$ \\
\hline
\end{tabular}


The initial stress distribution in the soil before load application is assumed to be undisturbed by the anchor installation process, e.g. horizontal and vertical stress components are considered to be equal. A typical load record without spectral filtering acting at the upper end of the cable is shown in Figure 4(a), while its corresponding frequency density function is depicted in Figure 4(b). As it may be observed, the mean load is approximately $\mathbf{f}_{\mathrm{b}} \approx 4000 \mathrm{kN}$ and associated main frequencies of load record are around $0.002 \mathrm{~Hz}$.

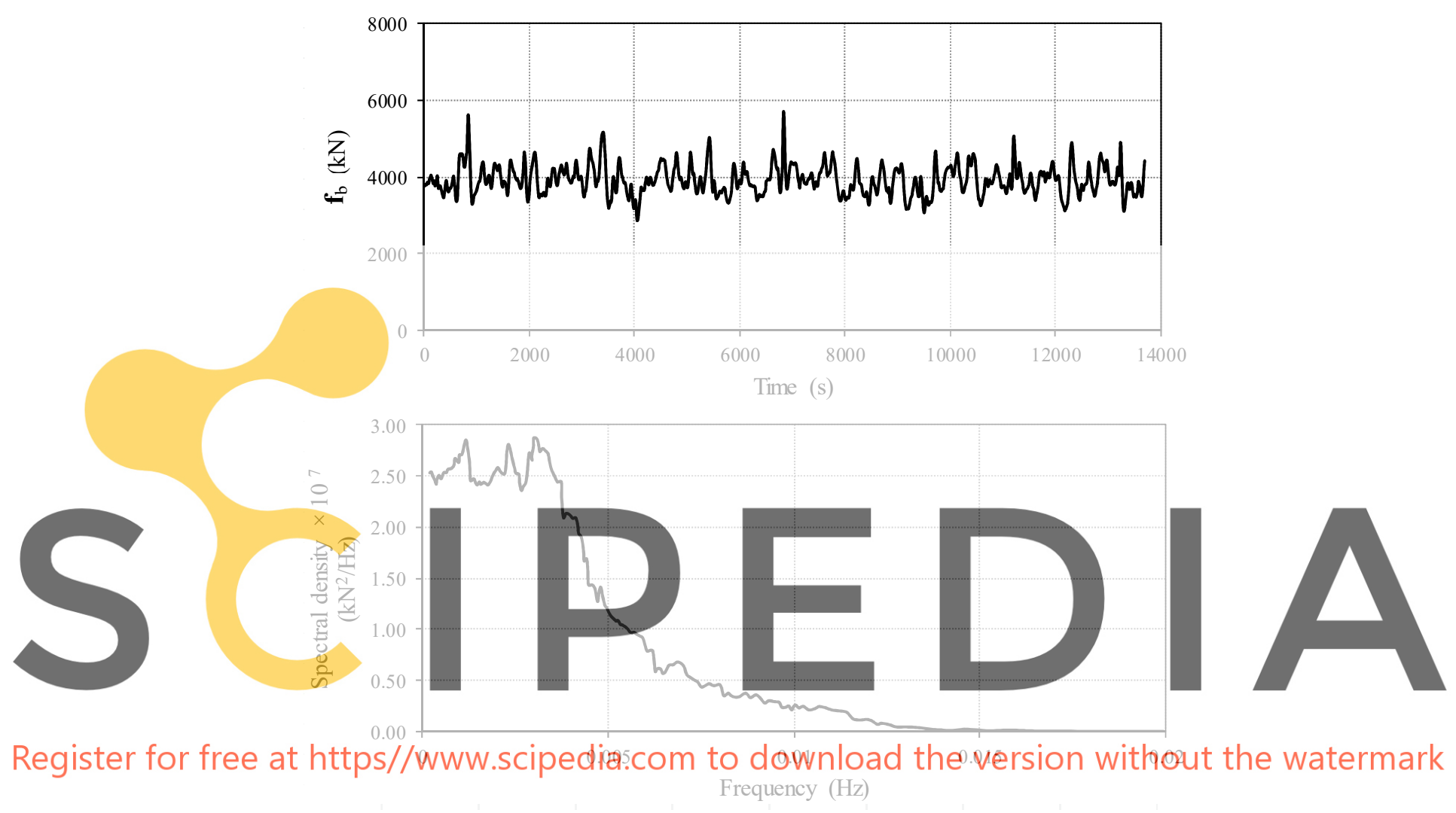

Figure 4: Load record: a) Force in time; b) Spectral density function

\subsection{Preliminary results}

Inertial effects can be introduced by the applied axial load to the mechanical system. To quantify this effect, free vibration responses are obtained by means of impulsive loads as illustrated in Figure 5. The loading process consists in applying a quasi-static axial load $\mathbf{f}_{\mathrm{b} 0}=$ $\mathbf{f}_{\mathrm{b}} / 4$ or $\mathbf{f}_{\mathrm{b} 0}=\mathbf{f}_{\mathrm{b}} / 2$ at the free end of the mooring cable, followed by impulsive loads $\Delta \mathbf{f}_{\mathrm{b}}=\mathbf{f}_{\mathrm{b} 0} /$ 2 or $\Delta \mathbf{f}_{\mathrm{b}}=\mathbf{f}_{\mathrm{b} 0} / 4$. The spectral density function of the numerical simulation is then displayed in Figure 6. As it may be observed, the main frequencies of the mechanical system are around $20 \mathrm{~Hz}$, which are considerably greater than those of the applied load in Figure 4(b). This preliminary result suggests that inertial effects could be neglected with safety from the analysis. 

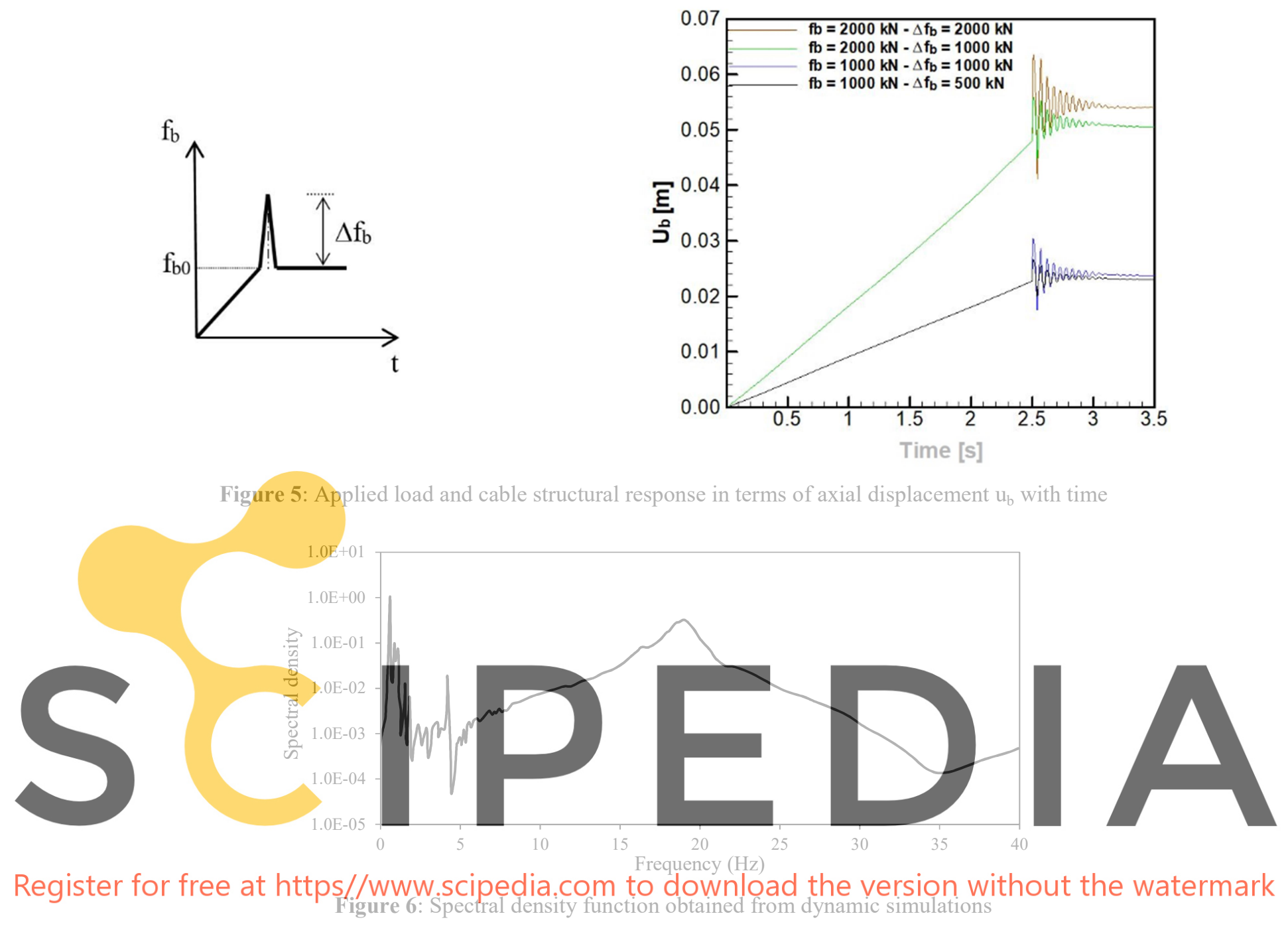

\subsection{Further Simulations}

The effect of anchoring depths on the evaluation of torpedo reaction is performed based on three configurations $\mathrm{h}=15 \mathrm{~m}, 20 \mathrm{~m}$ and $25 \mathrm{~m}$. The applied axial load $\mathbf{f}_{\mathrm{b}}$ is obtained from spectral filtering of load record depicted in Figure 4(a), which yields a smaller load record. Also, time duration of 1.2 seconds is preliminary adopted for numerical results since similar trends in response are expected for later times. Figure 7 displays evolution of torpedo reaction $\mathbf{f}_{\mathrm{t}}$ with time for different anchoring depths. As it may be observed, load attenuation increases with depth and corresponding percentages are summarized in Table 2. It may be concluded that an average load attenuation nearly $25 \%$ is obtained.

To study the effect of numerical integration in the overall response of the mechanical system, an additional analysis is carried out for the case of $15 \mathrm{~m}$ depth. In this case, the results of the hexahedral finite element with one-integration point, named stabilized element, are compared with those of the classical element in Figure 8. The classical element uses selective integration with full and reduced integration, respectively, for the deviatory and volumetric parts in the undrained situation. As it may be observed, the torpedo reaction $\mathbf{f}_{\mathrm{t}}$ correlates well 
with that of the stabilized element. However, the classical element is advantageous because it presents a robust behavior for nonlinear problems involving high gradients of plasticity, in which the stabilized elements could suffer from convergence problems. Although this is not the case in the current example, it can occur in other scenarios. Then, more parametric studies are needed to verify this situation.

Table 2: Load attenuation as function of anchoring depth

\begin{tabular}{ccc}
\hline Anchoring depth & Average attenuation & Attenuation at peak load \\
\hline $15 \mathrm{~m}$ & $20.7 \%$ & $23.5 \%$ \\
\hline $20 \mathrm{~m}$ & $24.6 \%$ & $26.4 \%$ \\
\hline $25 \mathrm{~m}$ & $26.8 \%$ & $27.9 \%$ \\
\hline
\end{tabular}

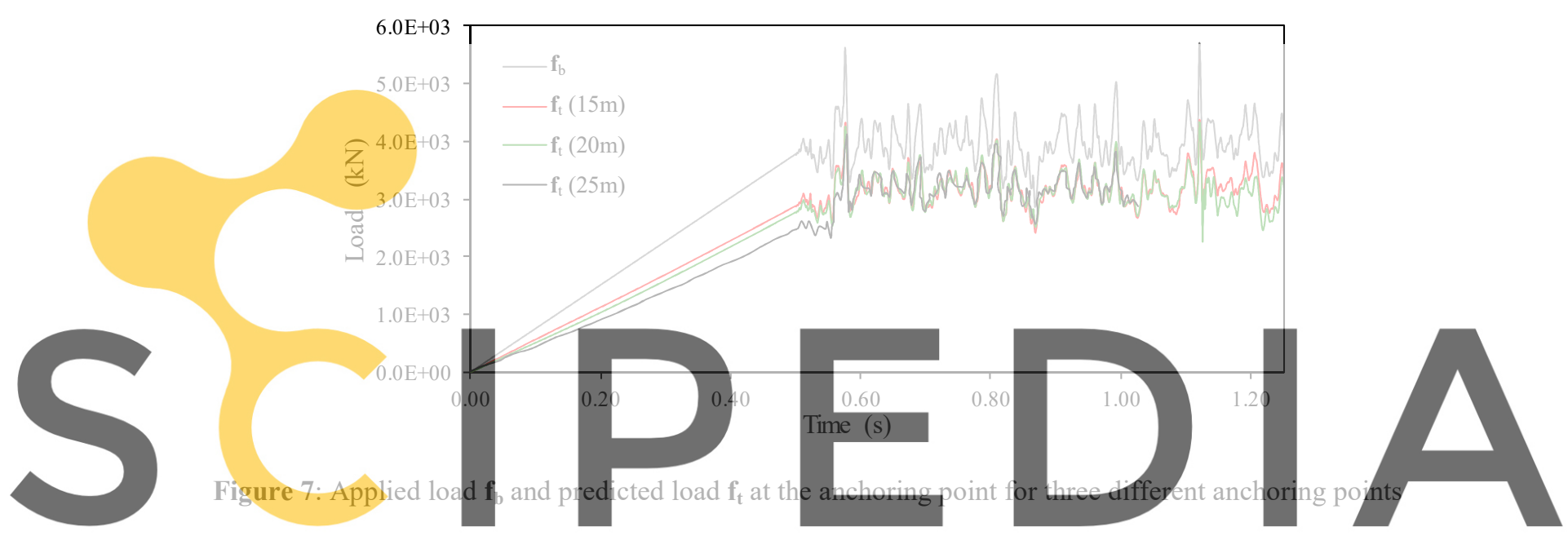

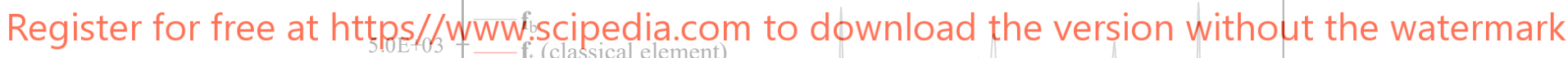

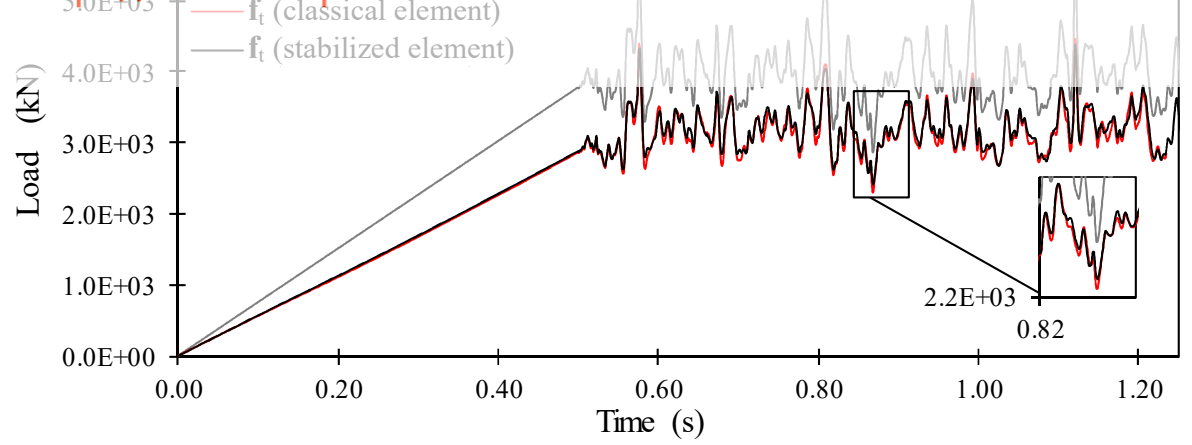

Figure 8: Applied load $\mathbf{f}_{\mathrm{b}}$ and predicted load $\mathbf{f}_{\mathrm{t}}$ at the anchoring point for different finite elements.

In relation to the size of the problem and computational effort, the smaller finite element mesh composed of 44800 hexahedral elements requires solving 149445 linear equations in each iteration. The common approach consisted in run a dynamic analysis ignoring inertial effects with at least 1200 steps for covering 1.2 seconds of load record, which gives a fictitious time step of 1.0E-03 seconds. A normal serial run in this condition can take seven 
days in an desktop computer with the following system properties, Intel (R) Core (TM) i5$2500 \mathrm{CPU} @ 3.3 \mathrm{GHz}$ and 3.49 of RAM. At the current stage of the project, various iterative solvers have been tested together with parallel directives using OpenMP to reduce computational time. Work is in progress in this aspect.

\section{CONCLUSIONS}

- Preliminary results indicate that a static analysis is suitable for the numerical simulation of the mechanical system composed of embedded mooring line and surrounding soil in the undrained condition. This is because the main frequencies of the load record are much smaller than those of the system.

- $\quad$ Load $\mathbf{f}_{\mathrm{b}}$ applied at the sea bed is attenuated around 25\% at the point in the anchoring device.

- Classical hexahedral finite elements with selective integration are shown to be competitive with one-point integration finite elements with stabilization. This is advantageous because classical elements are numerically more robust in problems involving high gradients of plasticity.

\section{REFERENCES}

[1] Zhou, Y.D. Cheuk, C.Y. and Tham, L.G. An embedded bond-slip model for finite modeling of soil-nail interaction. Computers and Geotechnics (2009) 36:1090-1097.

[2] Duarte, L.A. and Awruch, A.M. Geometrically nonlinear static and dynamic analysis of shells and plates using the eight-node hexahedral element with one-point quadrature. Finite Elements in Analysis and Design (2004) 40:1297-1315.

[3] Figuereido, M.P. Maghous, S. and Campos, A. Three-dimensional finite element analysis of reinforced concrete structural elements regarded as elastoplastic multiphase media. Materials and Structures (2013) 46:383-404.

[4] Yu, L. and Tan, J.H. Numerical investigation of seabed interaction in time domain analysis of mooring cables. Journal of Hydrodynamics (2006) 18:424-430.

[5] Wang, L. Guo, Z. and Yuan, F. Three-dimensional interaction between anchor chain and seabed. Applied Ocean Research (2010) 32:404-413.

[6] Braun, A.L. Bruch, A. and Maghous, S. A mixed 3D-1D finite element formulation for analysis of geomaterial structures with embedded curvilinear inclusions: application to load transfer in mooring anchor systems. Latin American Journal of Solids and Structures (2018) 15:1-36. 